\title{
Radio and gamma-ray constraints on dark matter annihilation in the Galactic center
}

\author{
R. M. Crocker ${ }^{1,2,3 *}$, N. F. Bell ${ }^{1 \dagger}$, C. Balázs ${ }^{2 \ddagger}$, and D. I. Jones ${ }^{3 \S}$ \\ ${ }^{1}$ School of Physics, University of Melbourne, 3010, Victoria, Australia \\ ${ }^{2}$ School of Physics, Monash University, 3800, Victoria, Australia \\ ${ }^{3}$ Max Planck Institut für Kernphysik, Saupfercheckweg 1, D-69117 Heidelberg, Germany
}

(Dated: August 26, 2018)

\begin{abstract}
We determine upper limits on the dark matter (DM) self-annihilation cross section for scenarios in which annihilation leads to the production of electron-positron pairs. In the Galactic centre (GC), relativistic electrons and positrons produce a radio flux via synchroton emission, and a gamma ray flux via bremsstrahlung and inverse Compton scattering. On the basis of archival, interferometric and single-dish radio data, we have determined the radio spectrum of an elliptical region around the Galactic centre of extent $3^{\circ}$ semi-major axis (along the Galactic plane) and $1^{\circ}$ semi-minor axis and a second, rectangular region, also centered on the GC, of extent $1.6^{\circ} \times 0.6^{\circ}$. The radio spectra of both regions are non-thermal over the range of frequencies for which we have data: $74 \mathrm{MHz}-10 \mathrm{GHz}$. We also consider gamma-ray data covering the same region from the EGRET instrument (about $\mathrm{GeV}$ ) and from HESS (around TeV). We show how the combination of these data can be used to place robust constraints on DM annihilation scenarios, in a way which is relatively insensitive to assumptions about the magnetic field amplitude in this region. Our results are approximately an order of magnitude more constraining than existing Galactic centre radio and gamma ray limits. For a DM mass of $m_{\chi}=10 \mathrm{GeV}$, and an NFW profile, we find $\left\langle\sigma_{\mathrm{A}} v\right\rangle \leq$ few $\times 10^{-25} \mathrm{~cm}^{3} \mathrm{~s}^{-1}$.
\end{abstract}

PACS numbers: 95.35.+d, 95.85.Bh, 98.70.Vc

\section{INTRODUCTION}

It is a remarkable fact that the identity of most of the matter in the Universe is unknown. An abundance of observational evidence allows us to infer the existence of dark matter [1-3] via its gravitational influence. However we have as yet no direct detection and very little information about its corpuscular properties. In this paper, we focus on one of the fundamental particle properties of dark matter: its self annihilation cross section.

If the dark matter were in thermal equilibrium in the early Universe, the annihilation cross section would determine the relic density in the Universe today. A velocity averaged annihilation cross section of $\left\langle\sigma_{A} v\right\rangle_{\text {th }} \sim$ few $\times 10^{-26} \mathrm{~cm}^{3} \mathrm{~s}^{-1}$ is required in order to produce the relic abundance of $\Omega_{\mathrm{DM}} \sim 0.2$. However, it is not necessary for DM to be a thermal relic, in which case both (significantly) larger or smaller cross sections are possible. Regardless of the thermal history, $\left\langle\sigma_{A} v\right\rangle$ controls the annihilation rate in DM halos in the Universe today, thus determining the size of detectable signals emanating from regions of DM concentration. It is these annihilation fluxes that potentially permit the technique of indirect detection of dark matter.

Recent cosmic ray positron/electron data from a number of experiments have led to much excitement about indirect DM detection. Anomalies in the cosmic ray positron spectra have been reported by the PAMELA,

\footnotetext{
*roland.crocker@mpi-hd.mpg.de

${ }^{\dagger}$ n.bell@unimelb.edu.au

${ }^{\ddagger}$ csaba.balazs@sci.monash.edu.au

§djones@mpi-hd.mpg.de
}

Fermi and HESS experiments, implying an apparent excess of positrons beyond those due to conventional astrophysical processes. PAMELA [4] has observed a rise in the $e^{+} /\left(e^{-}+e^{+}\right)$flux at energies above approximately 10 $\mathrm{GeV}$, while recent data from Fermi LAT [5] and HESS [6] show an excess in the $\left(e^{-}+e^{+}\right)$flux up to and beyond $1 \mathrm{TeV}$, respectively. (ATIC [7] and PPB-BETS [8] observed a similar excess, however, with considerably higher uncertainty than Fermi.)

The explanation of these positron excesses is far from clear, and, in light of these, researchers have been motivated to examine or re-examine conventional cosmic ray interaction [9] and propagation models [10, 11], acceleration of $e^{+} e^{-}$in cosmic ray sources [12-14], electron and positron emission from supernova remnants [15, 16], and the production of positrons by pulsars [17-24]. As an alternative to conventional astrophysical mechanisms, it has also been speculated that DM annihilation or decay in the Milky Way may be the source of the excess electrons and positrons.

Many authors have proposed models in which electron and positron fluxes arise from DM annihilation [2543] or decay [44-53], either directly or indirectly. (See also, Ref. [54] and references therein.) Note that, in contrast to the positron data, the PAMELA antiproton/proton observations do not indicate an anomalous contribution [57]. ${ }^{1} \quad$ Therefore, DM models which feature significant hadronic annihilation modes are constrained, while leptonic channels are preferred. How-

\footnotetext{
1 For completeness, we mention that both the reality of an anomaly in the PAMELA $e^{+} /\left(e^{-}+e^{+}\right)$flux and the absence of such in the anti-proton flux have been questioned $[55,56]$.
} 
ever, in order to account for the observed positron spectra, $\left\langle\sigma_{A} v\right\rangle$ must be significantly enhanced above the expected thermal relic value. Such an enhancement could be of astrophysical origin, e.g., a boost due to significant substructure throughout the halo or a local clump of dark matter [58, 59]. Alternatively, it could be of particle physics origin, e.g., a Breit-Wigner resonant enhancement [60-63] or the Sommerfeld effect (see, e.g., Refs. $[25,26,64,65])$ in which low velocity annihilation (i.e. in Galactic halos) is enhanced while early Universe freeze-out is affected to a lesser degree [66-69].

Several techniques may be used to constrain the production of $e^{+}$and $e^{-}$within Galactic halos, all of which rely on other accompanying observational signals. Charged particle production in a halo is necessarily accompanied by photon signals, including gamma rays, Xrays, microwaves and radio waves. These signals are produced via the various energy loss processes that charged particles undergo, examples of which include synchrotron emission in Galactic magnetic fields, inverse Compton scattering of electrons from interstellar radiation field, and bremsstrahlung. Charged particle production is also accompanied by internal bremsstrahlung radiation [7074], which is an electromagnetic radiative correction, and is not due to interaction in a medium.

In this work, we shall use the electron energy loss processes to derive constraints on $\left\langle\sigma_{A} v\right\rangle$. Annihilation channels we consider include both direct production of monoenergetic $e^{ \pm}$, and channels in which a spectrum of secondary $e^{ \pm}$are generated via decays of primary annihilation products, such as $\bar{q} q$. Note that, given their identical distributions and radiative signatures (at the energies under consideration), we shall imply both electrons and positron when we refer to "electrons" in this work, unless explicitly noted otherwise. Our analysis is distinguished from previous work in this area by the use of a new synthesis [75] of existing Galactic center radio data which allows us to derive particularly sensitive constraints. In addition, we make a careful study of the interplay of various energy loss processes, and show that a combination of different techniques leave our final bounds relatively insensitive to uncertainties in magnetic field amplitude.

A number of astrophysical uncertainties enter our calculations. As with all indirect detection analyses, we have a sensitivity to the assumed dark matter halo density profile. In addition we are subject to uncertainties in the Galactic magnetic field amplitude, the background light density, and the gas density. Variation in the assumed magnitudes of these parameters alters the proportion of electron cooling that takes place via the various energy loss processes. Higher magnetic field strengths, for example, lead to greater synchrotron losses, while higher background light density leads to more inverse Compton scattering. However, while the astrophysical assumptions control the relative importance of the various electron energy loss processes, the eventual total energy loss is a constant. (Note that, as we show below, relativistic electrons in the GC lose their energy - via whatever exact combination of processes - before they are transported out of the region.) Therefore, while constraints from a single process (say, synchrotron radiation) are individually quite uncertain, we show that the combined constraints derived using all energy loss processes are robust and feature only mild dependence on these astrophysical parameters.

This paper is structured as follows: in section II we discuss the radio and $\gamma$-ray data which provide the empirical constraints on the various DM models we test. We also describe the physical environment at the GC (magnetic field, ambient hydrogen number density) which determines how the cooling and subsequent radiation from relativistic electrons proceeds and the DM profiles we investigate. Section III describes the distribution of electrons putatively injected by DM annihilations and explains how this injection spectrum is shaped into a steady state distribution by the various cooling processes. We also set out here our calculation of the radio and $\gamma$-ray emission by these steady state electron distributions. In section IV we compare predicted emission from DM annihilation (parameterized in terms of DM particle mass and velocity averaged cross section) against empirical data and delineate the regions of parameter space we can thereby exclude. We also compare our results with bounds existing in the literature. Section V contains our concluding remarks.

\section{ASTROPHYSICAL INPUTS}

\section{A. Radio Data}

LaRosa et al. [76] observed a discrete but diffuse, nonthermal radio source (DNS) covering a roughly elliptical region around the Galactic centre (GC) of extent $3^{\circ}$ semimajor axis (along the Galactic plane) and $1^{\circ}$ semi-minor axis between 74 and $330 \mathrm{MHz}$. This angular region corresponds, more-or-less, to the usual definition of the Galactic nuclear bulge [77]. Subsequently, the work of Crocker et al. [75] assembled archival, interferometric and singledish radio data that demonstrates that the DNS radio structure is evident at frequencies up to $10 \mathrm{GHz}$ and has a non-thermal radio spectrum over the full $74 \mathrm{MHz}-$ $10 \mathrm{GHz}$ range of the data (see Fig. 1 for the spectrum and refer to Table I for references and other pertinent information on each radio datum). It should be noted though that, significantly for the purposes of Crocker et $a l$. , the spectrum of the DNS can not be described as a pure power law, but rather exhibits a spectral downbreak at about a GHz. For a full account of the radio data processing and analysis supporting this conclusion the reader should refer to [75], but we repeat a number of particularly relevant points below.

We also consider the constraints that arise from radio spectrum of the smaller region defined by $|l|<0.8^{\circ}$ and $|b|<0.3^{\circ}$. We refer to this as the HESS region, as it corresponds to a region for which the gamma ray intensity 
is reported by the HESS collaboration.

\begin{tabular}{|c|c|c|c|c|c|}
\hline \hline$\nu(\mathrm{GHz})$ & Telescope & Beam & Flux density & Error & Ref. \\
\hline $.074^{*}$ & VLA & $2^{\prime}$ & $16,200 \mathrm{Jy}$ & $1,000 \mathrm{Jy}$ & {$[76]$} \\
$.330^{\dagger}$ & Green Bank & 39 & $18,000 \mathrm{Jy}$ & $5 \%$ & {$[76]$} \\
1.408 & Effelsberg & $9.4^{\prime}$ & $7,300 \mathrm{Jy}$ & $10 \%$ & {$[78]$} \\
2.417 & Parkes & $10.4^{\prime}$ & $4,900 \mathrm{Jy}$ & $6 \%$ & {$[79]$} \\
2.695 & Effelsberg & $4.3^{\prime}$ & $4,400 \mathrm{Jy}$ & $10 \%$ & {$[80]$} \\
10.29 & Nobeyama & $2.9^{\prime}$ & $1,400 \mathrm{Jy}$ & $7 \%$ & {$[81]$} \\
\hline
\end{tabular}

TABLE I: Surveys and Observational data used to derive the spectrum for the $6^{\circ} \times 2^{\circ}$ region centred on the GC. Notes: *At $74 \mathrm{MHz}$ the large-angle Galactic plane synchrotron background/foreground flux contribution is not measured (and hence not accounted for) due to the interferometric nature of the VLA observation. 'Total flux supplied by Dr Crystal Brogan (private communication).

Radio data at $74 \mathrm{MHz}$ [76] were obtained by the Very Large Array (i.e., an interferometer), while data at all higher frequencies come from single dish instruments. (Because of the problem of free-free absorption along the line-of-sight to the GC [76] we do not employ the $74 \mathrm{MHz}$ datum as a constraint in our fitting below. At higher frequencies, because of the $\propto \nu^{-2.1}$ dependence of the free-free optical depth, absorption by this process is not a significant factor.) The radio flux at every frequency under consideration has or might have contributions from i) synchrotron emission by cosmic ray electrons along the line of sight, i.e., both in front of and behind the GC along the Galactic plane; ii) synchrotron emission by cosmic ray electrons gyrating in the GC itself; iii) discrete, astrophysical sources in the GC and along the line-of-sight (these include optically thin and optically thick thermal bremsstrahlung emitters and those with non-thermal spectra); iv) diffuse, large scale HII regions (i.e., regions of ionized atomic hydrogen that produce thermal bremsstrahlung); and v) (possibly and as investigated in this paper) relativistic electrons and positrons injected following annihilation or decay of putative DM particles.

As detailed in Ref. [75], the contribution of discrete sources (taken to be emission on angular scales $\lesssim 1.2^{\circ}$ ) can be measured in the single dish data by Fourier analysis. (At $330 \mathrm{MHz}$ the contribution of discrete sources was measured directly with the VLA [76]). This discrete source contribution is at a $<20 \%$ level at all frequencies under consideration and has been removed from every radio datum we use in our analysis. Otherwise, we remove no other astrophysical contribution and, in considering possible limits on synchrotron emission by relativistic electrons and positrons injected by DM annihilation or decay, we take the conservative approach [82] of requiring that predicted synchrotron emission be no larger than $3 \sigma$ above any radio datum.

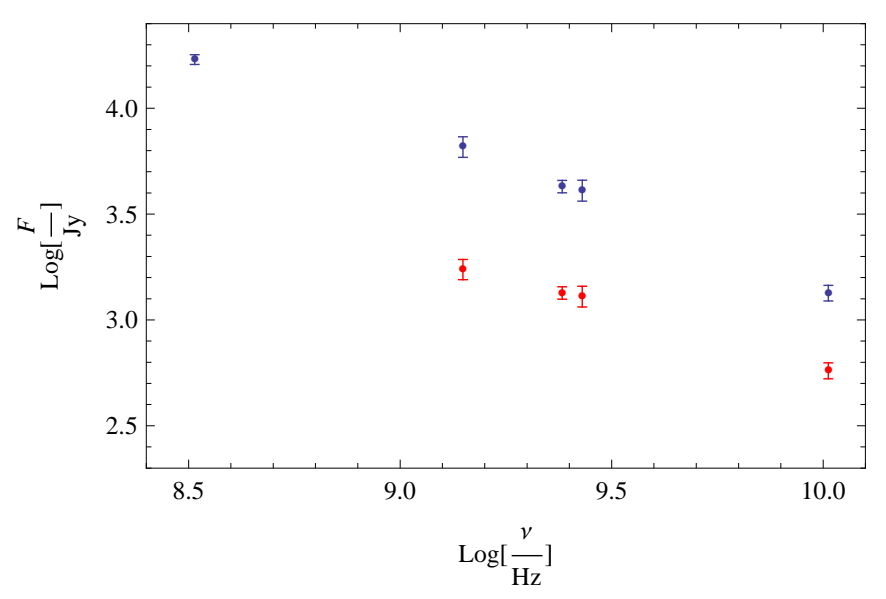

FIG. 1: Radio spectra of the DNS (blue, upper) and HESS (red, lower) regions.

\section{B. Gamma-Ray Data}

In addition to synchrotron radiation, bremsstrahlung and (sub-dominantly) inverse Compton (IC) emission will inescapably be generated by any relativistic electron population given the presence of background gas (mostly molecular hydrogen) and the GC's background light field. Electrons with GeV scale energies produce both bremsstrahlung photons of approximately $100 \mathrm{MeV}$ and synchrotron radiation at $\mathrm{GHz}$ frequencies (given the range of magnetic field amplitude that is physically plausible for the GC; see below). This means that $\gamma$-ray observations by the EGRET instrument (onboard the Compton Gamma-Ray Observatory) are particularly complementary to the radio data introduced above, as both signals are produced by electrons of the same energy. Finally, additional $\gamma$-ray photons are also generated from neutral meson decay in any scenario involving quark-pair production in DM annihilation and both $\bar{q} q$ and $e^{+} e^{-}$scenarios generate internal bremsstrahlung $\gamma$-rays.

\section{EGRET Data}

We claim a conservative upper limit to the integral gamma ray intensity from the DNS region. This is obtained from Figs. 2(c) and 2(d) of Hunter et al. [84] which, when combined, show a pedestal in the super-300 $\mathrm{MeV}$ intensity of $\sim 3 \times 10^{-4} \mathrm{~cm}^{-2} \mathrm{~s}^{-1} \mathrm{sr}^{-1}$, averaging over $|b|<2^{\circ}$ and within the longitude defined by $|l|<30^{\circ}$. Given that the DNS region is much smaller in extent (in longitude) than the pedestal, and that there are no structures evident in these longitude-dependent intensity plots on the scale of the DNS, we estimate an upper-limit to any exotic contribution to the intensity in the DNS region at the level of twice the largest actual excursion in the intensity above the pedestal value, viz. $1 \times 10^{-4} \mathrm{~cm}^{-2} \mathrm{~s}^{-1} \mathrm{sr}^{-1}$. We are confident that a 
more detailed analysis would produce a more stringent constraint. In any case, results pertaining to the region from the Fermi (GLAST) telescope are eagerly awaited.

\section{HESS Data}

Similarly, bremsstrahlung and IC emission by the higher energy members of the electron population will generate $\sim \mathrm{TeV}$ gamma rays (as will internal bremsstrahlung and, where applicable, neutral meson decay). The differential intensity in this energy range may be compared with the (conservative) upper limit obtained from observations by HESS [83]. This instrument has detected diffuse emission over the approximate energy range 300 $\mathrm{GeV}-10 \mathrm{TeV}$ and over the smaller solid angle defined by $|l|<0.8^{\circ}$ and $|b|<0.3^{\circ}$. The $\mathrm{TeV}$ intensity recorded is $1.4 \times 10^{-20} \mathrm{~cm}^{-2} \mathrm{eV}^{-1} \mathrm{~s}^{-1} \mathrm{sr}^{-1}$ with only limited and dimmer diffuse emission detected outside this region but within the DNS field. The HESS data analysis does, however, include a background subtraction from a nearby region, and this must be accounted for in our analysis[74, 85]. Because of this background subtraction, in the case of an NFW profile, a DM scenario may predict an absolute flux of $\gamma$-rays at $\mathrm{TeV}$ energies and within the HESS field up to $2 \times$ the observed $\sim \mathrm{TeV}$ intensity. For the isothermal profile, on the other hand, because of the very flat distribution and the consequently very small relative difference in $\gamma$-ray intensity predicted for the HESS region and the background region, no competitive constraint is proffered by the $\sim \mathrm{TeV}$ data at all (and we, therefore, do not plot $\mathrm{TeV} \gamma$-ray constraint curve for this profile).

\section{Ambient Environment}

\section{Gas Density}

Over the DNS region, the volumetric-average hydrogen number density can be calculated to be about $13 \mathrm{~cm}^{-3}$ on the basis of the data presented in Ref. $[77]^{2}$. Much of this density can be ascribed to molecular hydrogen found at very high number density $\left(>10^{3.5} \mathrm{~cm}^{-3}\right.$ ) but small filling factor $(<0.01)$ in the cores of the unusually-dense, Galactic center molecular cloud population $[77,86]$. Quite possibly electrons impinging from outside the dense molecular material may be excluded from it [87-89]. Excluding this very high density phase $\left(n_{H}>10^{3} \mathrm{~cm}^{-3}\right)$ but including hydrogen in relatively low density molecular

\footnotetext{
${ }^{2}$ We assume the DNS volume to be an elliptical spheroid with circular cross-section in the Galactic plane (i.e., its greatest lineof-sight extent is equal to its width along the plane). This implies a total volume of $\sim 3.0 \times 10^{63} \mathrm{~cm}^{3}$.
}

hydrogen, atomic hydrogen (HI) and 'warm' ionized hydrogen $\left(\mathrm{H}^{+}\right)$one finds a minimum (path-integrated) $n_{H}$ for a DNS electron of $\sim 2.7 \mathrm{~cm}^{-3}$ and $\sim 23 \mathrm{~cm}^{-3}$. We adopt these values in our calculations henceforth. (The putative 'very hot', plasma phase of the DNS, that would have a filling factor of $\sim 85 \%$, would have lower number density than this. However, relativistic electrons would not be trapped in this phase [90] and, in any case, the existence of this phases has recently been thrown into considerable doubt: see below.)

\section{Galactic Magnetic Field}

The amplitude of the GC magnetic field remains uncertain by about two orders of magnitude.

- On the basis of the 74 and $330 \mathrm{MHz}$ observations of the DNS (nuclear bulge) introduced above, LaRosa et al. [76] use the equipartition argument to suggest that the field on DNS size scales is only around $6 \mu \mathrm{G}$ - very similar in amplitude to that typical for the Galactic disk - climbing to only about 10 $\mu \mathrm{G}$ over the inner $1 .^{\circ} 6$ along the Galactic plane. (Coincidentally, the region over which the HESS collaboration measures a diffuse $\sim \mathrm{TeV} \gamma$-ray intensity [83].) These smaller field amplitudes have recently received support from rotation measures to external galaxies close to the GC [91].

- A rather stronger field over the DNS region is suggested by the work of Spergel and Blitz [92] who note that the putative "very hot" $(8 \mathrm{keV}) \mathrm{X}$-ray emitting plasma found throughout the GC [93] and the unusually dense and turbulent molecular gas might be in pressure equilibrium (implying near equipartition between these ISM phases). In analogy with conditions local to the solar system [94], it might then be expected that the magnetic field also contribute a roughly equal pressure requiring it to have about $100 \mu \mathrm{G}$ amplitude. The existence of the very hot plasma has recently been cast into extreme doubt, however, with the apparently diffuse X-ray emission being, it is contested [95], essentially ascribable to unresolved, point X-ray sources. This would essentially nullify the Spergel and Blitz argument.

- Finally, (coincidentally or not) over the HESS field, radio observations of the 'non thermal filaments' (synchrotron-emitting radio structures, unique to the inner Galaxy, that run essentially perpendicular to the Galactic plane) suggest that the ambient field might be as strong as $1 \mathrm{mG}$ [96-98].

The situation regarding the GC magnetic field is entirely unclear, therefore.

Recently this argument has been joined by Crocker et al. [75]. On the basis of a simultaneous analysis of the higher-frequency part of the radio spectrum of the 
DNS radio structure and $\sim \mathrm{GeV} \gamma$-ray data covering the same region (i.e., the very data described above), these authors have recently claimed a probable value for the magnetic field in this region of $100 \mu \mathrm{G}$ with a lower limit (at $95 \%$ confidence) at $50 \mu \mathrm{G}$. However, we cannot selfconsistently assume this result holds for the purposes of the current paper. The analysis in Crocker et al. assumes that the observed radio emission from the DNS region can be attributed to "conventional" astrophysical electrons governed by a pure power law at injection, and that any spectral features detected in the observed spectrum arise in the cooling of the injection spectrum (which is initially featureless). If we allow for the possibility that DM makes an additional contribution to the electron injection spectrum, the kinematics of DM annihilation allows for the possibility of spectral features in the injection spectrum of the in situ electron population, so the assumption of Crocker et al. that cooling alone introduces spectral features does not necessarily hold. (E.g., one can find a fit acceptable at better than $2 \sigma$ confidence to the radio spectrum invoking synchrotron emission by a population of relativistic electrons injected following the annihilation of dark matter particles with a mass $\sim 30 \mathrm{GeV}$ and assuming the quark hadronization spectrum given below. We certainly do not claim this as a detection!) This implies we cannot self-consistently adopt the magnetic field strength inferred in Ref. [75] in this work.

Given the uncertainties explained above, in this paper we investigate DM constraints across the whole range of magnetic field amplitudes supported by the existing literature, which we take to be $10-100 \mu \mathrm{G}$ for the DNS region and $10-1000 \mu \mathrm{G}$ for the HESS diffuse flux region defined above. However, as we demonstrate below, radio and $\gamma$-ray data offer complimentary constraints over this range: radio data provide the more severe restriction on $\left\langle\sigma_{A} v\right\rangle$ for strong fields, while $\gamma$-ray data are more constraining for weak fields (because, in the latter case, there is less synchrotron suppression of the high-energy electrons required for IC up-scattering of ambient light into $\sim \mathrm{GeV}$ and $\sim \mathrm{TeV}$ energy ranges).

\section{Dark matter distribution}

We employ the usual Navarro-Frank-White (NFW) distribution [99]

$$
\rho(r)=\frac{\rho_{\mathrm{h}}}{\frac{r}{r_{\mathrm{h}}}\left(1+\frac{r}{r_{\mathrm{h}}}\right)^{2}},
$$

where $\rho_{\mathrm{h}}=0.572 \mathrm{GeV} \mathrm{cm}^{-3}$ and $r_{\mathrm{h}}=14 \mathrm{kpc}$. The above implies a volumetric-average DM density of $3.6 \times 10^{10}$ $\mathrm{eV} \mathrm{cm}{ }^{-3}$ over the DNS region and $1.2 \times 10^{11} \mathrm{eV} \mathrm{cm}^{-3}$ over the HESS region. We employ these volume-average quantities in our calculations (given we assume a onezone model).
We also investigate limits in the case that the DM distribution follows a truncated isothermal profile. Here we assume the parametrization presented in Ref. [82] following Ref. [100]:

$$
\rho(r)=\frac{\rho_{\mathrm{s}}}{1+\left(\frac{r}{r_{\mathrm{s}}}\right)^{2}},
$$

where $\rho_{\mathrm{s}}=1.16 \mathrm{GeV} \mathrm{cm}^{-3}$ and $r_{\mathrm{s}}=5 \mathrm{kpc}$.

\section{DARK MATTER SIGNALS}

\section{A. Dark matter annihilation and primary electron spectrum}

In typical models with weakly-interacting massive particles (WIMPs), the DM masses fall in the GeV-TeV range, while the DM annihilation cross section is given approximately by the thermal relic value of $\left\langle\sigma_{A} v\right\rangle_{\text {th }} \sim$ few $\times 10^{-26} \mathrm{~cm}^{3} \mathrm{~s}^{-1}$. However, the DM self annihilation cross section can be much smaller if coannihilations play a significant role in determining the WIMP freezeout density, as occurs in many supersymmetric DM scenarios. Conversely, the Sommerfeld effect can boost the self annihilation cross section relevant for galactic halos in the Universe today, effectively resulting in values much larger than the thermal relic expectation. Moreover, if dark matter was populated via some non-thermal mechanism, $\left\langle\sigma_{A} v\right\rangle_{\text {th }}$ is not relevant to the presently observed average abundance. It is thus appropriate to take $\left\langle\sigma_{A} v\right\rangle$ to be a free parameter, spanning a wide range of possible values. Likewise, we shall also take the DM mass to be a free parameter. (Indeed, masses above $1 \mathrm{TeV}$ have been considered in light of recent cosmic ray positron results and data from Fermi LAT.) We shall consider two DM annihilation processes: 1. $\chi \chi \rightarrow \bar{q} q$ and 2. $\chi \chi \rightarrow e^{+} e^{-}$.

$$
\text { 1. } \chi \chi \rightarrow \bar{q} q
$$

Annihilation to $\bar{q} q$ is taken as a representative prototype of many DM models, as the resulting electron energy spectrum is a good approximation to that for many annihilation channels. For example, the annihilation spectrum for a specific supersymmetric WIMP candidate may be calculated with the DarkSUSY package [101]. However, the annihilation channels $\chi \chi \rightarrow \bar{q} q, Z Z, W^{+} W^{-}$ all lead to essentially the same electron energy spectrum (while the electron energy spectrum resulting from $\chi \chi \rightarrow \tau^{+} \tau^{-}$is significantly different). Note that for annihilation to $Z Z, W^{+} W^{-}$, and $\bar{q} q$, hadronization or decay of the primary annihilation products leads, via charged pions and muon intermediaries, to positrons and electrons with a broad spectrum of energies.

For simplicity, we shall assume full annihilation into $\bar{q} q$. For this channel, $e^{-}$and $\left(e^{+}\right)$will be produced by decaying muons (anti-muons) produced in charged pion 
decay. The resulting $e^{+}+e^{-}$spectrum can be written, following Borriello et al. [102], in a simple polynomial form of the ratio $E_{e} / m_{\chi} c^{2}$ :

$$
\frac{\mathrm{d} N_{e}}{\mathrm{~d} E_{e}}\left(E_{e}\right)=\frac{2}{m_{\chi} c^{2}} \sum_{j \in J} a_{j}\left(\frac{E_{e}}{m_{\chi} c^{2}}\right)^{j},
$$

where $J=\left\{-\frac{3}{2},-\frac{1}{2}, 0, \frac{1}{2}, 2,3\right\}$ and the coefficients $a_{j}$ are listed in Table II taken from [102].

This analytical expression is a reasonable approximation for $E_{e} \gtrsim 1 \mathrm{GeV}$. At low energies the analytical expression from [102] has an asymptotic behaviour $\propto E_{e}^{-1.5}$ while the kinematics of the charged meson and muon decay chains mean that the actual spectrum has a lowenergy cut-off. Because of the large magnetic fields we sample, our calculated radio flux from synchrotron emission is potentially sensitive to this effect at the lowest frequencies considered (cf. [102]). We therefore explicitly introduce a cut-off by patching on a numerical description of the low-energy spectrum we have previously obtained [87] in the context of a numerical treatment of secondary electron production from hadronic cosmic ray interactions (where identical kinematical considerations come into play) to Borriello et al.'s distribution.

TABLE II: $a_{j}$ values from [102]

\begin{tabular}{|c|c|}
\hline coefficient & numerical \\
\hline$a_{-3 / 2}$ & 0.456 \\
$a_{-1 / 2}$ & -5.37 \\
$a_{0}$ & 10.9 \\
$a_{1 / 2}$ & -6.77 \\
$a_{2}$ & 0.969 \\
$a_{3}$ & -0.185 \\
\hline
\end{tabular}

$$
\text { 2. } \chi \chi \rightarrow e^{+} e^{-}
$$

We shall also consider direct annihilation to monoenergetic electrons, for which the electron energy spectrum per annihilation is simply given by

$$
\frac{\mathrm{d} N_{e}}{\mathrm{~d} E_{e}}\left(E_{e}\right)=2 \delta\left(m_{\chi}-E_{e}\right) .
$$

Note that the injection electron spectra differ from the steady state electron spectra due to the action of electron cooling, as discussed below.

\section{B. Steady-state electron distribution}

The injection spectrum of electrons produced in the Galaxy, at position $r$, as a result of Dark Matter annihilation is

$$
Q\left(E_{e}, r\right)=\frac{1}{2}\left(\frac{\rho(r)}{m_{\chi}}\right)^{2}\left\langle\sigma_{\mathrm{A}} v\right\rangle \frac{\mathrm{d} N_{e}}{\mathrm{~d} E_{e}} .
$$

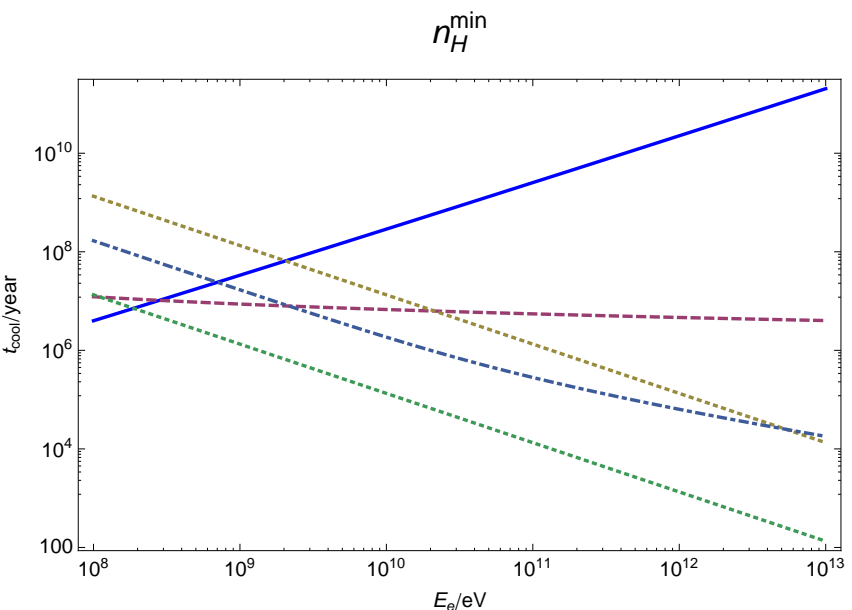

FIG. 2: Cooling time-scale due to various processes affecting electrons and positrons in the GC environment as a function of energy. Curves are - solid: ionization, dashed: bremsstrahlung; dotted: synchrotron for (upper) $10 \mu \mathrm{G}$ and (lower) 100 $\mu \mathrm{G}$; and dot-dashed: inverse Compton.

In general, the injected electrons loose energy interacting with the interstellar medium and move away from the production site. Both these broad processes lead to modifications of the shape of the injection spectrum. If, however, the transport timescale is much longer than the cooling timescale for electrons in the relevant energy range one is in the so-called "thick-target" regime in which case the steady-state (cooled) electron distribution can be written as

$$
\frac{d n_{e}}{d E_{e}}\left(E_{e}, \vec{r}\right)=\frac{\int_{E_{e}}^{m_{\chi} c^{2}} d E_{e}^{\prime} Q\left(E_{e}^{\prime}, \vec{r}\right)}{-d E_{e}\left(E_{e}\right) / d t}
$$

where $d E_{e}\left(E_{e}\right) / d t$ is the cooling rate, resulting from the sum of several energy loss processes that affect electrons (described immediately below). We show in the Appendix that we are justified in adopting the thick target regime for the GC environment.

\section{Electron cooling processes}

In the GC environment four cooling processes are potentially important for charged leptons with energies such that they radiate at radio and $\gamma$-ray wavelengths (electrons and positrons suffer essentially identical energy losses over the relevant energy scale and are treated identically). These processes are dominantly (from low to high energy) ionization, bremsstrahlung, and synchrotron and/or IC emission. We show the cooling timescales, as a function of electron (or positron) energy, in Fig. 2 for plausible GC environment parameters. In Fig. 3 we show examples of the shape of the injected electron (and positron) spectrum together with the steadystate electron distribution after cooling. 
Injection vs Cooled Spectrum

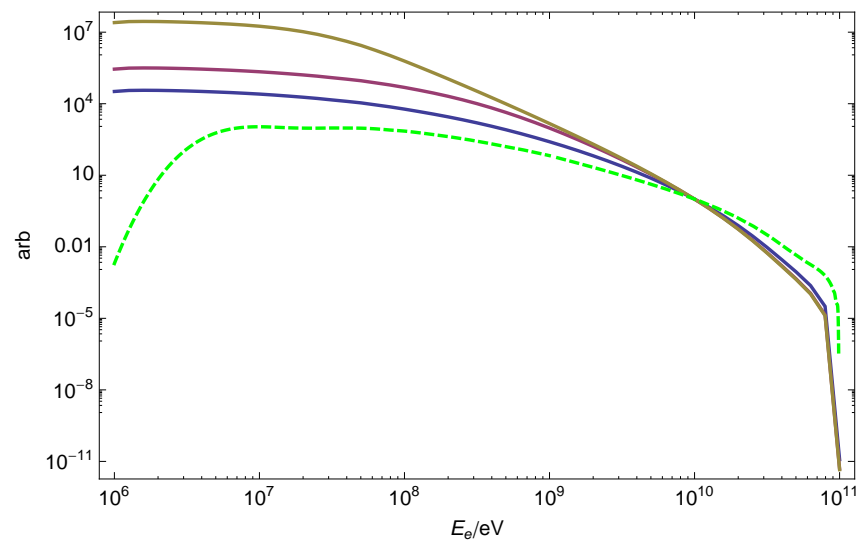

FIG. 3: Shape of the injected electron + positron distribution (green; dashed) and steady state distributions (for various magnetic field amplitudes - mG (yellow; solid, upper); 100 $\mu \mathrm{G}$ (purple; solid, center); and $10 \mu \mathrm{G}$ (blue; solid, lower). The curves have been normalized to 1 (on an arbitrary scale) at an energy of $10 \mathrm{GeV}$. Note the low-energy, kinematic cutoff in the injected distribution. The plot assumes $m_{\chi}=100$ $\mathrm{GeV}$.

\section{Radiative Processes}

Of the cooling processes listed above, all except ionization lead to potentially-observable signatures at radio and $\gamma$-ray wavelengths. The amount of cooling that takes places via either radio or gamma ray emission - and the constraints derived on the basis of that channel - depend on assumptions made about the astrophysical inputs. We calculate separate constraints from radio and gamma ray emission, in each case choosing astrophysical inputs conservatively. In particular, for the hydrogen density, $n_{H}$, we conservatively choose a value which leads to smallest observational signal for each process considered. The magnetic field strength is left as a free parameter to be varied, and we shall demonstrate the our final results are relatively insensitive to this parameter. In contrast, we fix the ambient hydrogen number density to be the appropriate minimal value set out in $\S \mathrm{II} \mathrm{C} 1$ (given the consideration that most of the ambient gas is 'locked-up' in molecular cloud cores of very high density but extremely low filling factor). We describe our treatment of each radiative process below. Representative $\gamma$-ray spectra from each of the relevant process (for a $m_{\chi}=1 \mathrm{TeV}$ DM mass and $\left\langle\sigma_{\mathrm{A}} v\right\rangle=10^{-26} \mathrm{~cm}^{3} \mathrm{~s}^{-1}$, with a Borriello et al. [102] $e^{ \pm}$spectrum) are shown in fig.4.

\section{Synchrotron}

We use standard formulae [103] to calculate the radio emissivity of relativistic electrons (and positrons) in the Galactic center magnetic field.

\section{Bremsstrahlung Calculation}

We use standard formulae (see Ref. [104], p. 84 et seq.) to calculate the bremsstrahlung emissivity of relativistic electrons (and positrons) in the Galactic center environment.

\section{Inverse Compton Calculation}

For inverse Compton cooling and emission calculations we assume the full interstellar radiation field determined for the inner $500 \mathrm{pc}$ of the Galaxy in [105]. This has an energy density around $19 \mathrm{eV} \mathrm{cm}^{-3}$. We calculate the Compton scattering emissivity of relativistic electrons using the full Klein-Nishina cross section set out in section 2.3.3 of Ref. [106]. For the minimal $n_{H}$ value we assume, the integrated energy flux $(100 \mathrm{MeV}+)$ from IC emission is about $70 \%$ as large as the bremsstrahlung energy flux.

\section{Gamma-rays from neutral meson decay}

For DM annihilation into $q \bar{q}$ as investigated by Borriello et al., a $\gamma$-ray signal from the decay of neutral mesons produced in quark hadronization is inescapable. The contribution of this process is often dominant for relevant energies and environmental parameters (see fig. 4). We employ a parameterization of the numerical results of Bergström et al.[107] for the $\gamma$-ray spectra produced by quark hadronization following annihilation of $500 \mathrm{GeV}$ neutralinos (displayed in the their figure 12 and parameterized in terms of the scaling variable $x \equiv E_{\gamma} / m_{\chi}$ ). A representative distribution is that following $u \bar{u}$ production:

$$
\frac{d N_{\gamma}}{d x}=\exp (-6.8 x) x^{-1.5}
$$

We also assume a branching ratio of $100 \%$ into the maximally massive quark pair allowable given the DM mass (which means either $b \bar{b}$ or $t \bar{t}$ over the range of $m_{\chi}$ we explore).

\section{Gamma-rays from internal bremsstrahlung}

DM annihilation to any charged particles is necessarily accompanied by internal bremsstrahlung (IB) radiation [70-74]. This is an electromagnetic radiative correction, and not to be confused with the regular bremsstrahlung considered in this paper. IB produces hard gamma rays up to the dark matter mass, with an approximately model-independent spectrum, largely free from both particle and astrophysics uncertainties. For the (sub-dominant) contribution of internal bremsstrahlung 


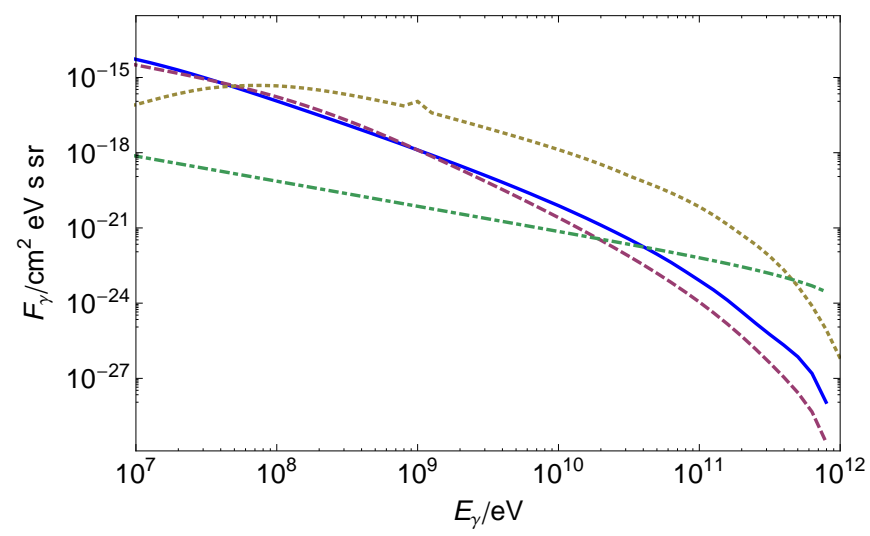

FIG. 4: Sample $\gamma$-ray spectra of the DNS region for a $m_{\chi}=1$ TeV DM mass and $\left\langle\sigma_{\mathrm{A}} v\right\rangle=10^{-26} \mathrm{~cm}^{3} \mathrm{~s}^{-1}$, with a Borriello et al. [102] $e^{ \pm}$spectrum, a plausible ambient field of 100 $\mu \mathrm{G}$, and an ambient hydrogen number density of $\sim 3 \mathrm{~cm}^{-3}$. Curves are: (blue, solid) inverse Compton; (purple,dashed) bremsstrahlung; (yellow,dotted) neutral meson decay (following hadronization); and (green, dot-dash) internal bremsstrahlung.

we employ the parameterization of Refs. [71, 74]:

$$
\frac{d N_{\gamma}}{d E_{\gamma}}=\frac{\alpha}{\pi E_{\gamma}}\left[\ln \left(\frac{s^{\prime}}{m_{e}^{2}}\right)-1\right]\left[1+\left(\frac{s^{\prime}}{s}\right)^{2}\right],
$$

where $s=4 m_{\chi}^{2}$ and $s^{\prime}=4 m_{\chi}\left(m_{\chi}-E\right)$. (This equation has to modified for the case of $q \bar{q}$ production by replacing the electron mass with the effective quark mass, and $\alpha$ with $Q_{q}^{2} \alpha$, where $Q_{q}$ is the electric charge of quark $q[72]$.)

\section{DM ANNIHILATION CONSTRAINTS}

\section{A. Discussion of results}

Figures 5, 6, 7 and 8, display the bounds we obtain for the annihilation cross section, as a function of DM mass. We show results corresponding to our four main scenarios, namely, the $\chi \chi \rightarrow \bar{q} q$ and $\chi \chi \rightarrow e^{+} e^{-}$processes, for both NFW and isothermal DM halo profiles. For each scenario, we show how the constraints depend upon the assumed magnetic field strength within the plausible range $10-100 \mu \mathrm{G}$.

Neglecting the $\sim \mathrm{TeV} \gamma$-ray constraints for the moment, in each scenario investigated $\gamma$-ray data is the most constraining at low magnetic field amplitude, and radio the best at high. Adjusting the magnetic field amplitude alters the proportion of electron cooling that occurs via synchrotron emission. Thus, for low (high) $B$ amplitude the radio flux is less (more) constraining. The total energy loss of the electron population is controlled by a combination of the available cooling mechanisms, such that reducing the amount of cooling taking place by synchrotron emission leads to relatively more gamma rays emission via bremsstrahlung and inverse Compton scattering. (Note that any individual electron must eventually loose all its energy via one mechanism or another, and it is impossible for this energy loss to occur without the production of detectable signals.) Therefore while the radio constraints, considered alone, are quite sensitive to the assumed magnetic field strength, our final results, being the strongest of either the radio or gamma constraints at a given $B$ amplitude, are much less sensitive to the assumed value of $B$.

For the NFW profile, we find that the $\sim \mathrm{TeV} \gamma$-ray constraint invariably becomes the best for larger values of $m_{\chi}$. (As remarked above, because of the background subtraction procedure, $\mathrm{TeV} \gamma$-ray data does not proffer a competitive constraint for relatively flat profiles like the isothermal). The $\sim \mathrm{TeV} \gamma$-ray constraint curve is highly structured as a result of it being a comparison between predicted and observed differential $\gamma$-ray intensities at the nine separate energies the HESS collaboration has listed starting at $\sim 300 \mathrm{GeV}$ in concert with the three (for $e^{+} e^{-}$) of four (for $\bar{q} q$ ) $\gamma$-ray production mechanisms and their different thresholding effects with respect to the $m_{\chi}$ parameter. A somewhat similar effect - though less pronounced is see in the radio constraints. In contrast, the EGRET $\gamma$-ray constraints (taken above a single photon energy of $300 \mathrm{MeV}$ in the integral intensity) define a fairly smooth constraint for each scenario whereas .

In Fig. 9 we compare the best constraint obtained (from any modality except for $\sim \mathrm{TeV} \gamma$-rays) for plausible magnetic field values, namely 10, 30, 100, 1000 $\mu \mathrm{G}$. We see that the best constraint on $\langle\sigma v\rangle$ varies by a factor of $<10$ as $B$ is varied from $10-100 \mu \mathrm{G}$. (This is to be compared with a larger variation in the radio constraint alone of $\sim 30$.) Therefore, when considered in totality, the $\gamma$-ray and radio data conspire to generate constraints on DM mass/annihilation cross section that are roughly constant across the reasonable range of astrophysical parameters (in particular, magnetic field amplitude). For larger magnetic field amplitude (i.e. $B=1000 \mu \mathrm{G}$, which is at the upper end of the astrophysically plausible range) synchrotron emission dominates the cooling, and somewhat stronger constraints are obtained. This occurs because the field has become strong enough that the $\mathrm{GHz}$ range synchrotron-radiation is sampling lower energy (and therefore more numerous) electrons.

Comparing the figures for the NFW and isothermal profiles (e.g. Fig. 5 with Fig. 7, or Fig. 6 with Fig. 8) it is evident that the exclusion curves for the NFW profile are always much more constraining than for the corresponding isothermal scenario. This is due to the high central DM density of the strongly peaked NFW profile, in comparison with the low central DM density of the flatter isothermal profile

Likewise, the radio observation of the HESS region (small solid angle at the GC) provide more stringent con- 


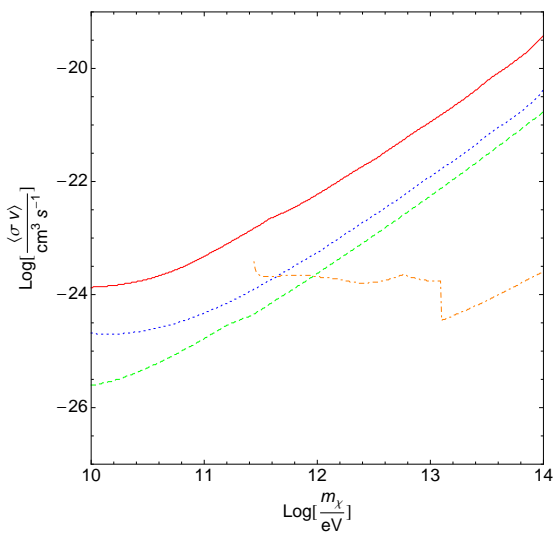

(a) $\chi \chi \rightarrow \bar{q} q$; NFW profile; $B=10 \mu \mathrm{G}$.

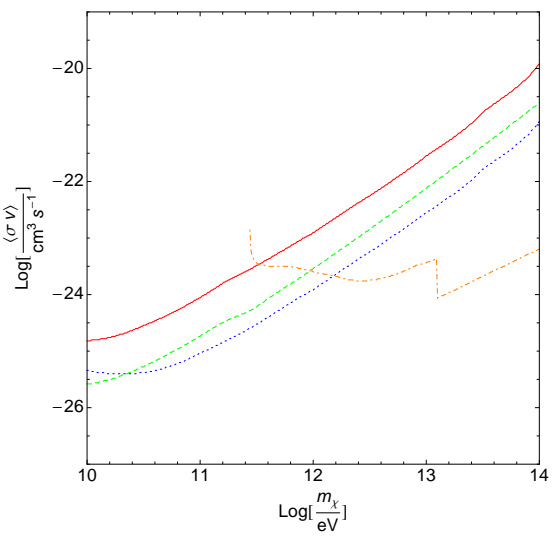

(b) $\chi \chi \rightarrow \bar{q} q$; NFW profile; $B=30 \mu \mathrm{G}$.

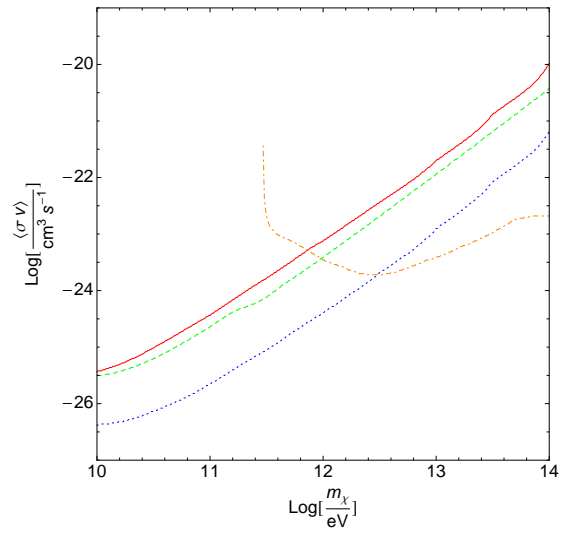

(c) $\chi \chi \rightarrow \bar{q} q$; NFW profile; $B=100 \mu \mathrm{G}$.

FIG. 5: Exclusion contours for the DM annihilation cross section assuming a $\chi \chi \rightarrow \bar{q} q$ annihilation channel (with a Borriello et al. [102] $e^{ \pm}$spectrum) and an NFW DM profile, obtained from DNS radio data (red, solid), HESS region radio data (blue, dotted), DNS region $\sim 300 \mathrm{MeV} \gamma$-ray data (green, dashed), and HESS region $\sim \mathrm{TeV} \gamma$-ray data (orange, dot-dashed). Relevant $\gamma$-ray production processes are neutral meson decay, bremsstrahlung, inverse Compton emission, and internal bremsstrahlung. Results are displayed for three Galactic magnetic field amplitudes within a plausible range (a) $10 \mu \mathrm{G}$, (b) $30 \mu \mathrm{G}$ and (c) 100 $\mu \mathrm{G}$.

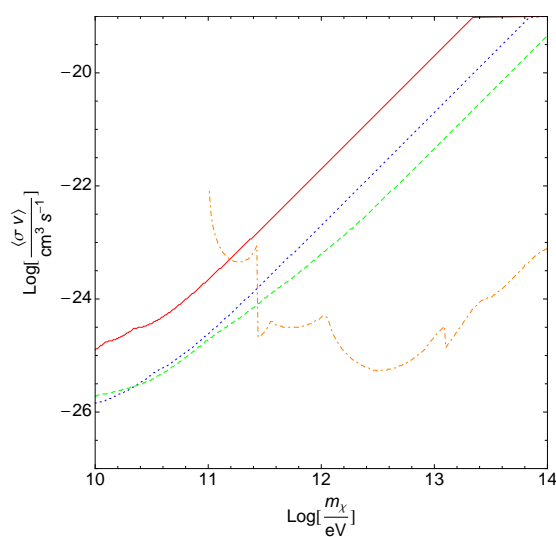

(a) $\chi \chi \rightarrow e^{+} e^{-}$; NFW profile; $B=10 \mu \mathrm{G}$.

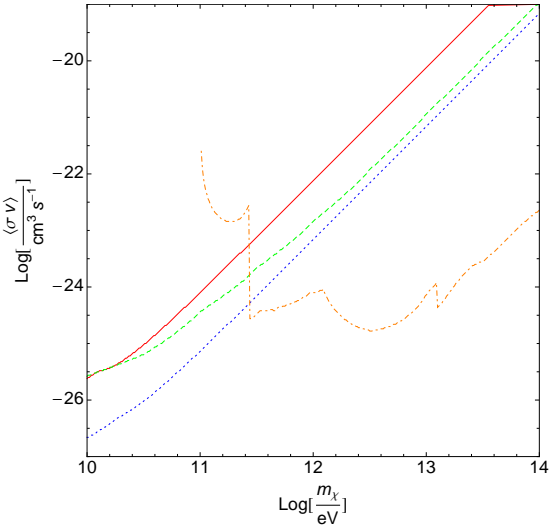

(b) $\chi \chi \rightarrow e^{+} e^{-}$; NFW profile; $B=30 \mu \mathrm{G}$.

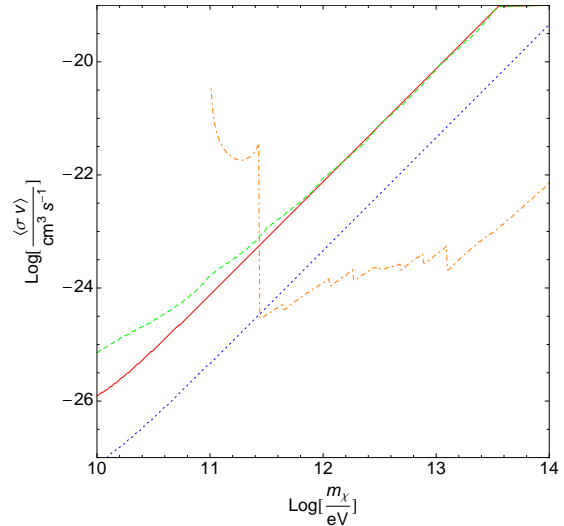

(c) $\chi \chi \rightarrow e^{+} e^{-}$; NFW profile; $B=100 \mu \mathrm{G}$.

FIG. 6: Exclusion contours for the DM annihilation cross section, assuming a $\chi \chi \rightarrow e^{+} e^{-}$annihilation channel (mono-energetic $e^{ \pm}$spectrum) and an NFW DM profile, obtained from DNS radio data (red, solid), HESS region radio data (blue, dotted), DNS region $\sim 300 \mathrm{MeV} \gamma$-ray data (green, dashed), and HESS region $\sim \mathrm{TeV} \gamma$-ray data (orange, dot-dashed). Relevant $\gamma$-ray production processes are bremsstrahlung, inverse Compton emission, and internal bremsstrahlung. Results are displayed for Galactic magnetic field amplitudes (a) $10 \mu \mathrm{G}$, (b) $30 \mu \mathrm{G}$ and (c) $100 \mu \mathrm{G}$.

straints than the radio observations of the DNS region (larger solid angle) for the peaked NFW profile. For the isothermal profile, however, we have something of the opposite situation: the radio bounds for the DNS regions are often more constraining than those for the HESS region. This can be understood since, for the flat isothermal profile, the average DM density is approximately the same for both regions, while the radio background for the DNS region is lower than for the HESS region.

With the exception of the HESS $\sim \mathrm{TeV} \gamma$-ray constraint, the $\bar{q} q$ injection scenario tends to generate somewhat tighter constraints than the monoenergetic $e^{+} e^{-}$ spectrum, for all values of $m_{\chi}$ larger than $\sim 100 \mathrm{GeV}$. In the $\bar{q} q$ case, electrons and positrons are injected as secondaries from meson decay following quark hadronization. Therefore, a single $\chi \chi \rightarrow \bar{q} q$ interaction produces more electrons and positrons (though of much lower energy) than for the monoenergetic $\chi \chi \rightarrow e^{+} e^{-}$case, and thus generally tighter limits. In addition, the production of $\gamma$-rays from neutral meson decay, leads to strong $\mathrm{MeV}$ gamma ray limits in the case of $\bar{q} q$. At the lowest energies (below $\sim 100 \mathrm{GeV}$ ), the $e^{+} e^{-}$radio limits surpass those for $\bar{q} q$, due to the higher average energy of the injection spectrum. 


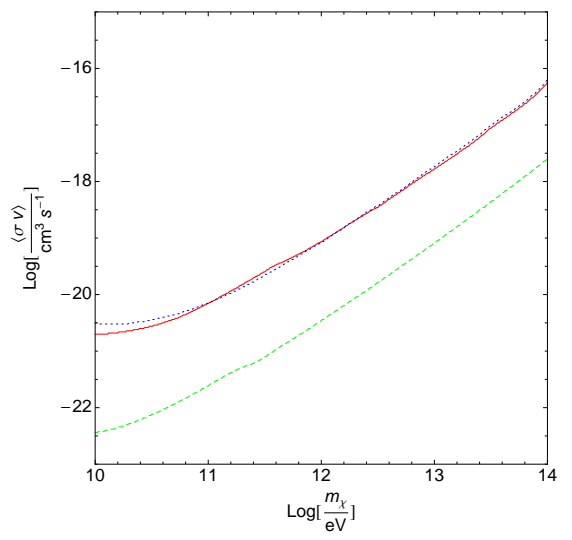

(a) $\chi \chi \rightarrow \bar{q} q$; Isothermal profile; $B=10 \mu \mathrm{G}$.

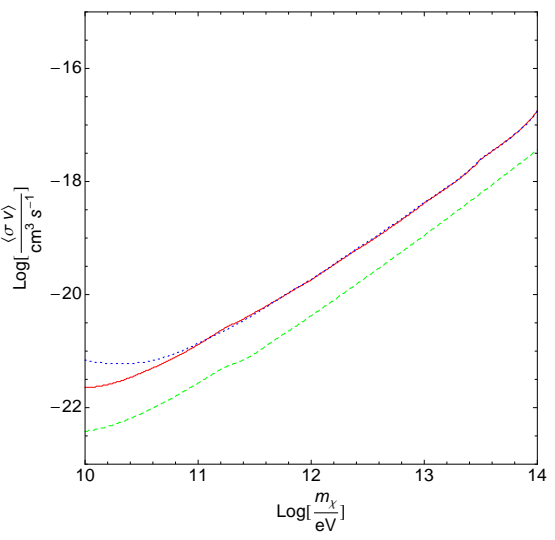

(b) $\chi \chi \rightarrow \bar{q} q$; Isothermal profile; $B=30 \mu \mathrm{G}$.

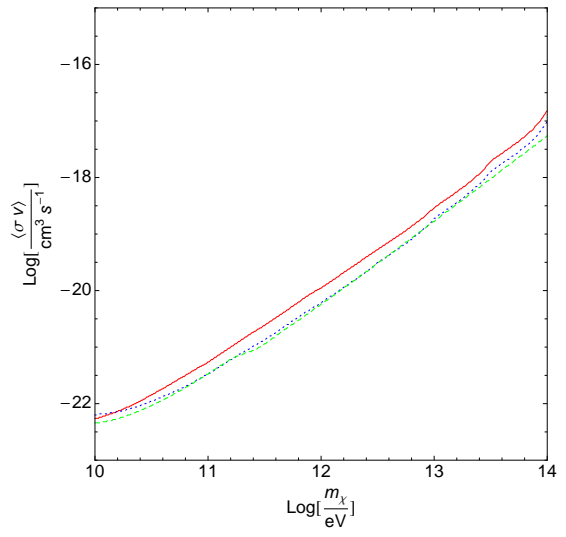

(c) $\chi \chi \rightarrow \bar{q} q$; Isothermal profile; $B=100 \mu \mathrm{G}$.

FIG. 7: Exclusion contours for the DM annihilation cross section assuming a $\chi \chi \rightarrow \bar{q} q$ annihilation channel (with a Borriello et al. [102] $e^{ \pm}$spectrum) and an Isothermal DM profile, obtained from DNS radio data (red, solid), HESS region radio data (blue, dotted), and $\sim 300 \mathrm{MeV} \gamma$-ray data (green, dashed). [For such a flat profile the $\sim \mathrm{TeV}$ data do not offer a competitive constraint: see the text.] Results are displayed for Galactic magnetic field amplitudes (a) $10 \mu \mathrm{G}$, (b) $30 \mu \mathrm{G}$ and (c) $100 \mu \mathrm{G}$.

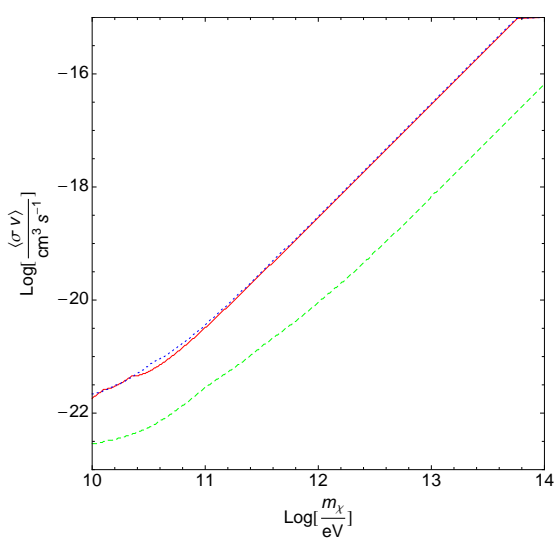

(a) $\chi \chi \rightarrow e^{+} e^{-}$; Isothermal profile; $B=10 \mu \mathrm{G}$.

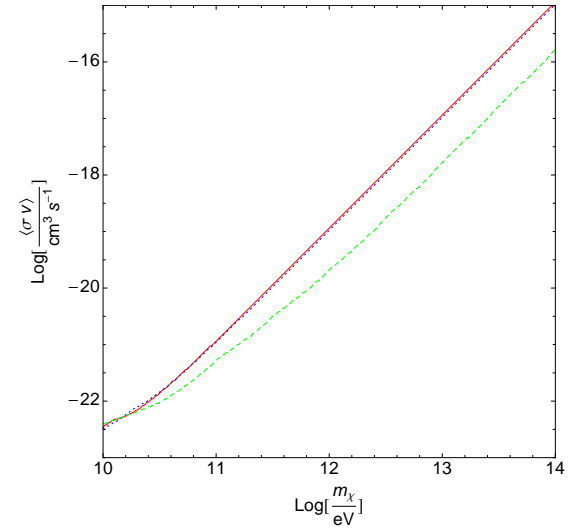

(b) $\chi \chi \rightarrow e^{+} e^{-}$; Isothermal profile; $B=30 \mu \mathrm{G}$.

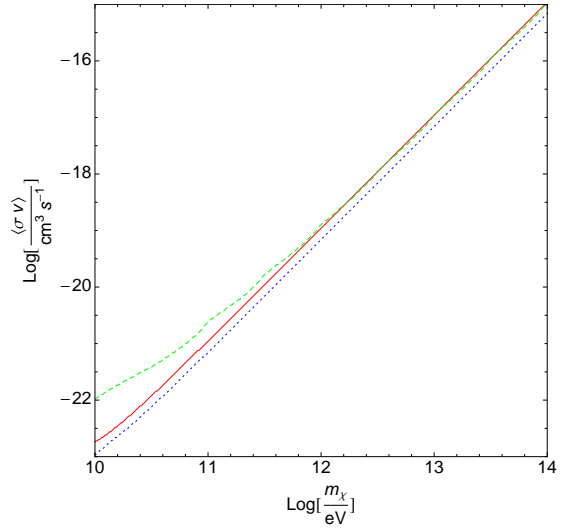

(c) $\chi \chi \rightarrow e^{+} e^{-}$; Isothermal profile; $B=100 \mu \mathrm{G}$.

FIG. 8: Exclusion contours for the DM annihilation cross section assuming a $\chi \chi \rightarrow e^{+} e^{-}$annihilation channel (mono-energetic $e^{ \pm}$spectrum) and an Isothermal DM profile, obtained from DNS radio data (red, solid), HESS region radio data (blue, dotted), and $\sim 300 \mathrm{MeV} \gamma$-ray data (green, dashed). [For such a flat profile the $\sim \mathrm{TeV}$ data do not offer a competitive constraint: see the text.] Results are displayed for Galactic magnetic field amplitudes (a) $10 \mu \mathrm{G}$, (b) $30 \mu \mathrm{G}$ and (c) $100 \mu \mathrm{G}$.

\section{B. Comparison with previous results}

A number of previous works have considered DM annihilation corss section constraints obtained from Galactic radio and $\gamma$-ray data. While our analysis is complementary to these prior studies, distinguished by the use of different GC radio data, it is useful to compare the strength of the limits obtained on $\langle\sigma v\rangle$ :

1. Of recent work, the most immediately comparable to ours - as discussed above - is that of Borriello et al. [102] whose NFW profile and electron injection spectrum we adopt (for the $\chi \chi \rightarrow \bar{q} q$ process). Our results are at least one order of magnitude and up to two orders of magnitude better than those ob- tained by these authors. The constraints of Borriello et al. are obtained from consideration of allsky radio data at frequencies between $100 \mathrm{MHz}$ and $23 \mathrm{GHz}$. These authors, however, specifically exclude a $15^{\circ} \times 15^{\circ}$ patch of sky centered on the GC (because their calculations are performed in the limit that only synchrotron cooling is important in shaping the steady state electron distribution and - as discussed at length above - in the higher gas densities near the GC, this will no longer be true given the consequent importance of ionization and bremsstrahlung).

2. Hooper [108] has considered constraints obtainable from WMAP data [109] at 22, 33, and $61 \mathrm{GHz}$. 


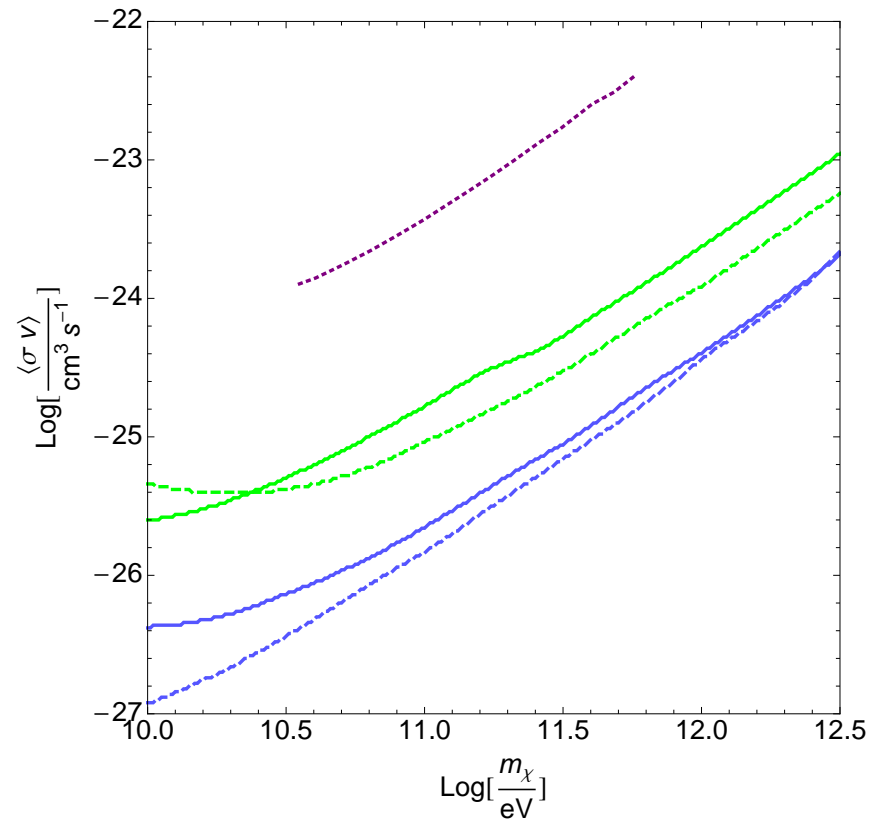

FIG. 9: Plot of the best constraint we find (from any modality except fot $\sim \mathrm{TeV} \gamma$-rays) over plausible magnetic field values, assuming a $\chi \chi \rightarrow \bar{q} q$ annihilation channel and an NFW DM profile. Curves are: green $-\gamma$-ray constraints for (dashed) 30 $\mu \mathrm{G}$ and (solid) $10 \mu \mathrm{G}$; and blue - radio constraints from the HESS region for (solid) $100 \mu \mathrm{G}$ field and (dashed) $1 \mathrm{mG}$ field. Also shown for comparison is the best constraint obtained by Borriello et al. [102] for this scenario, purple, dotted.

His analysis relies on astrophysical foregroundsubtracted data that putatively reveals a residue, unexplained "haze" [110] at all three WMAP frequencies on scales out to about $20^{\circ}$ from the GC (and absent elsewhere). The Hooper analysis, then, is intrinsically less conservative than the radio analysis presented here where we do not attempt such subtraction (except for discrete sources in the field which can be unambiguously identified on angular size grounds). While Hooper's constraints are particularly strong, some of the astrophysical assumptions adopted may be too optimistic. In particular he assumes a constant $10 \mu \mathrm{G}$ field and constant $5 \mathrm{eV} \mathrm{cm}^{-3}$ interstellar radiation field energy density over the $30^{\circ}$ angular region he investigates, the former being rather stronger and the latter rather weaker (at least towards the GC) than assumed by other authors [102, 111]. Grajek et al. [111] consider the same (foreground-subtracted) WMAP data investigated by Hooper. Assuming a rather weaker (and spatially-varying) magnetic field and a rather smaller ratio $U_{B} /\left(U_{B}+U_{\text {rad }}\right) \sim 0.1$, these authors find correspondingly weaker constraints on the annihilation cross section, which we surpass. (Note that the $W^{+} W^{-}$annihilation channel considered by Hooper and Grajek results in a very similar electron injection spectrum to the $\bar{q} q$ channel we consider.)

3. Finally, Bertone et al.[82] and Regis and Ullio [112] both also consider constraints arising from multiwavelength observations of the GC, including radio and $\gamma$-rays. These papers tend to focus on observations on much smaller angular scales around the super massive black at the Galactic dynamical center than investigated here. While some of the constraints (and projected constraints) derived are quite strong, on these small scales it is necessary to assume a particular model for the evolution of the magnetic field intensity (and the matter density controlling bremsstrahlung and ionization cooling). Such constraints are also particularly sensitive to the DM density at extremely small radii, which is highly uncertain.

We now compare with constraints derived using other techniques. For hadronic annihilation modes, constraints arising from the antiproton observations have been shown to be of comparable sensitivity to existing Galactic centre gamma ray and radio limits, e.g. Refs. [113, 114], for some models. For the monoenergetic $e^{+} e^{-}$annihilation channel, pure IB constraints were derived in Ref. [74]. For the NFW profile, the IB constraints are very strong for $10^{3} \lesssim m_{\chi} \lesssim 10^{4}$ (these arise from HESS gamma ray data near the GC) whereas our new radio/gamma ray bounds are better at lower mass. (For an isothermal profile, the IB constraints arising from the HESS data would be considerable weaker.) Note that for masses above $\sim 10 \mathrm{TeV}$, bounds on the total annihilation cross section to all final states, derived using neutrino data, become most constraining [115-117]. For higher masses, $m_{\chi} \gtrsim 10^{2}$, TeV the unitarity bound on the total cross section becomes the most restrictive constraint [118].

Turning now to non-Galactic based techniques, a number of authors have recently considered constraints arising from annihilation at high redshift. For example, Refs. [119-123] consider the effect of energy deposited by DM annihilation during the the reionization and recombination epochs. (Note that these techniques are complementary to those we consider here, being subject to quite different assumptions and systematics.) For an NFW profile and an $e^{+} e^{-}$annihilation channel the reionization limits are broadly comparable to the constraints we have derived here, with the boost factor required to be $\lesssim 10$ at $m_{\chi} \sim 100 \mathrm{GeV}$.

\section{SUMMARY AND CONCLUSIONS}

We have considered DM annihilation in the Galactic center, for scenarios in which annihilation leads to the production of relativistic electrons and positrons. Using signals arising from electron energy loss processes, namely synchrotron emission, bremsstrahlung and inverse Compton scattering (and, where appropriate, those 
due to neutral meson decay and internal bremsstrahlung), we have derived robust constraints on the velocityaveraged DM self annihilation cross section $\langle\sigma v\rangle$. The processes considered were $\chi \chi \rightarrow e^{+} e^{-}$which produces monoenergetic $e^{ \pm}$, and $\chi \chi \rightarrow \bar{q} q$, in which a spectrum of $e^{ \pm}$are generated via the charged pion decay chain. (The constraints for the later channel are expected to be very similar to those for other interesting final states, such as $W^{+} W^{-}$and $Z Z$.)

We have demonstrated that a combination of radio and gamma ray bounds is relatively insensitive to the assumed magnetic field amplitude within a plausible range, with the constraint on $\langle\sigma v\rangle$ varying by a factor of $<10$ as $B$ is varied from $10-100 \mu \mathrm{G}$. (Taken alone, the radio and gamma rays bounds are individually quite sensitive to the assumed magnetic field amplitude.) Our analysis is distinct from previous work in this area by the use of a new synthesis of Galactic center radio data assembled in Ref. [75].

Our constraints on velocity-averaged DM annihilation cross sections are conservative as we (i) do not remove (known) astrophysical contributions to the radio emission and (ii) do not include a contribution to the total radio emission from DM annihilation electrons and positrons along the line-of-sight but out of the GC.

Despite these conservative assumptions, our results are at least one order of magnitude and up to two orders of magnitude better than the most directly comparable previous limit (obtained by Borriello et al. for the same DM distributions and electron injection spectrum, but considering all-sky radio fluxes). Moreover, our constraints rule out a sizable portion of the parameter space that has been invoked to explain the various positron anomalies. (See, e.g. Refs. $[122,124]$ for recent determinations of the PAMELA/Fermi perferred regions.)

For an NFW profile and a $\chi \chi \rightarrow \bar{q} q$ annihilation process, we find the allowed boost factor is $\lesssim 1$ at $10 \mathrm{GeV}$ and $\lesssim 100$ at $1 \mathrm{TeV}$. For $\chi \chi \rightarrow e^{+} e^{-}$, the allowed boost factor is $<1$ at $10 \mathrm{GeV}$ and $<1000$ at $1 \mathrm{TeV}$. Note that these constraints apply to boost factors generated via whatever mechanism, be it a Breit-Wigner resonance, the Sommerfeld effect, or an enhancement due to DM substructure $^{3}$.

\footnotetext{
3 An interesting exception is the case where we can not reliably extrapolate between the local DM annihilation rate and that in the Galactic center, e.g., because of a variation in the dispersion velocity [125] or a local clump of dark matter.
}

\section{Acknowledgments}

The authors are indebted to T. Jacques, A. Mazumdar, N. Sahu, and F. Wang for invaluable discussions on various aspects of dark matter models and the indirect detection of dark matter signals. This research was funded in part by the Australian Research Council under Project ID DP0877916 and DP0988343.

\section{Appendix A: Electron transport}

Much research concerning cosmic ray electrons adopts the approach of taking measured or inferred values of the "diffusion coefficient" (for cosmic ray electrons at a particular energy), assuming some energy scaling for this quantity, and then deriving characteristic distances over which electrons might diffuse over their cooling times. There are many uncertainties attendant upon this statistical approach, particularly for the Galactic center environment where evidence points to ambient magnetic fields stronger and more turbulent than found typically in the disk. We prefer to take the approach of referring to direct modeling (by solving the Lorentz force equation) of individual particle trajectories for the GC environment. Here we rely on the work of Wommer et al. [126] who consider proton propagation in this environment. From the work of these authors we infer that $\mathcal{O}(\mathrm{TeV})$ energy protons might travel $\lesssim 0.2^{\circ}$ or 30 pc (at the GC distance) over their radiative lifetimes for an environment with a $10 \mu \mathrm{G}$ ambient field and $n_{H}=100 \mathrm{~cm}^{-3}$. In this environment the $\mathrm{TeV}$ proton cooling timescale (due to ionization and $p-p$ collisions) is $\sim 4 \times 10^{5}$ years. In contrast, for $\mathrm{GeV}$ scale electrons (synchrotron-radiating at about $\mathrm{GHz}$ frequencies), the cooling timescale is less than this at $\lesssim 2 \times 10^{5}$ years for the weakest magnetic field $(10 \mu \mathrm{G})$ and most tenuous gas environment $\left(n_{H} \simeq 3\right.$ $\mathrm{cm}^{-3}$ ) we consider. Given that both $\mathrm{TeV}$ protons and $\mathrm{GeV}$ electrons are ultra-relativistic and that diffusion distance will decline towards lower particle energies, we infer from Ref. [126] that $\mathrm{GeV}$ electrons will travel well less than $0.2^{\circ}$ or $30 \mathrm{pc}$ in their radiative lifetimes. Given this angular scale is well smaller than both the DNS and HESS fields we conclude that it is safe to conduct our calculations in the thick target limit.
[1] G. Jungman, M. Kamionkowski and K. Griest, Phys. Rept. 267, 195 (1996).

[2] G. Bertone, D. Hooper and J. Silk, Phys. Rept. 405, 279 (2005).

[3] L. Bergstrom, Rept. Prog. Phys. 63, 793 (2000).

[4] O. Adriani et al. [PAMELA Collaboration], Nature 458,
607 (2009) [arXiv:0810.4995 [astro-ph]].

[5] A. A. Abdo et al. [The Fermi LAT Collaboration], Phys. Rev. Lett. 102, 181101 (2009) [arXiv:0905.0025 [astroph.HE]].

[6] F. Aharonian et al.[HESS Collaboration], arXiv:0905.0105 [astro-ph.HE]. 
[7] J. Chang et al., Nature 456, 362 (2008).

[8] S. Torii et al. [PPB-BETS Collaboration], arXiv:0809.0760 [astro-ph].

[9] L. Stawarz, V. Petrosian and R. D. Blandford, arXiv:0908.1094 [astro-ph.GA].

[10] R. Cowsik and B. Burch, arXiv:0905.2136 [astroph.CO].

[11] B. Katz, K. Blum and E. Waxman, arXiv:0907.1686 [astro-ph.HE].

[12] P. Blasi, arXiv:0903.2794 [astro-ph.HE].

[13] H. B. Hu, Q. Yuan, B. Wang, C. Fan, J. L. Zhang and X. J. Bi, arXiv:0901.1520 [astro-ph].

[14] S. Dado and A. Dar, arXiv:0903.0165 [astro-ph.HE].

[15] N. J. Shaviv, E. Nakar and T. Piran, arXiv:0902.0376 [astro-ph.HE].

[16] Y. Fujita, K. Kohri, R. Yamazaki and K. Ioka, arXiv:0903.5298 [astro-ph.HE].

[17] D. Hooper, P. Blasi and P. D. Serpico, JCAP 0901, 025 (2009) [arXiv:0810.1527 [astro-ph]].

[18] H. Yuksel, M. D. Kistler and T. Stanev, arXiv:0810.2784 [astro-ph].

[19] S. Profumo, arXiv:0812.4457 [astro-ph].

[20] D. Malyshev, I. Cholis and J. Gelfand, arXiv:0903.1310 [astro-ph.HE].

[21] V. Barger, Y. Gao, W. Y. Keung, D. Marfatia and G. Shaughnessy, arXiv:0904.2001 [hep-ph].

[22] D. Grasso et al. [FERMI-LAT Collaboration], arXiv:0905.0636 [astro-ph.HE].

[23] P. Mertsch and S. Sarkar, arXiv:0905.3152 [astroph.HE].

[24] D. Malyshev, arXiv:0905.2611 [astro-ph.HE].

[25] M. Cirelli, M. Kadastik, M. Raidal and A. Strumia, Nucl. Phys. B 813, 1 (2009) [arXiv:0809.2409 [hep-ph]].

[26] N. Arkani-Hamed, D. P. Finkbeiner, T. R. Slatyer and N. Weiner, Phys. Rev. D 79, 015014 (2009) [arXiv:0810.0713 [hep-ph]].

[27] I. Cholis, D. P. Finkbeiner, L. Goodenough and N. Weiner, arXiv:0810.5344 [astro-ph].

[28] R. Harnik and G. D. Kribs, Phys. Rev. D 79, 095007 (2009) [arXiv:0810.5557 [hep-ph]].

[29] R. Allahverdi, B. Dutta, K. Richardson-McDaniel and Y. Santoso, arXiv:0812.2196 [hep-ph].

[30] X. Calmet and S. K. Majee, arXiv:0905.0956 [hep-ph].

[31] S. Shirai, F. Takahashi and T. T. Yanagida, arXiv:0905.0388 [hep-ph].

[32] C. H. Chen, C. Q. Geng and D. V. Zhuridov, arXiv:0905.0652 [hep-ph].

[33] K. Hamaguchi, K. Nakaji and E. Nakamura, arXiv:0905.1574 [hep-ph].

[34] N. Okada and T. Yamada, arXiv:0905.2801 [hep-ph].

[35] H. Fukuoka, J. Kubo and D. Suematsu, arXiv:0905.2847 [hep-ph].

[36] Y. Bai, M. Carena and J. Lykken, arXiv:0905.2964 [hep$\mathrm{ph}$.

[37] S. Shirai, F. Takahashi and T. T. Yanagida, arXiv:0905.3235 [hep-ph].

[38] C. H. Chen, arXiv:0905.3425 [hep-ph].

[39] J. Mardon, Y. Nomura and J. Thaler, arXiv:0905.3749 [hep-ph];

[40] D. A. Demir, L. L. Everett, M. Frank, L. Selbuz and I. Turan, arXiv:0906.3540 [hep-ph].

[41] D. Hooper and T. M. P. Tait, arXiv:0906.0362 [hep-ph].

[42] K. Y. Choi and C. E. Yaguna, arXiv:0906.0736 [hep-ph].

[43] D. Feldman, Z. Liu, P. Nath and B. D. Nelson,
arXiv:0907.5392 [hep-ph].

[44] P. f. Yin, Q. Yuan, J. Liu, J. Zhang, X. j. Bi and S. h. Zhu, Phys. Rev. D 79, 023512 (2009) [arXiv:0811.0176 [hep-ph]].

[45] K. Hamaguchi, E. Nakamura, S. Shirai and T. T. Yanagida, Phys. Lett. B 674, 299 (2009) [arXiv:0811.0737 [hep-ph]].

[46] A. Ibarra and D. Tran, JCAP 0902, 021 (2009) [arXiv:0811.1555 [hep-ph]].

[47] E. Nardi, F. Sannino and A. Strumia, JCAP 0901, 043 (2009) [arXiv:0811.4153 [hep-ph]].

[48] K. Ishiwata, S. Matsumoto and T. Moroi, Phys. Rev. D 79, 043527 (2009) [arXiv:0811.4492 [astro-ph]].

[49] S. De Lope Amigo, W. Y. Cheung, Z. Huang and S. P. Ng, JCAP 0906, 005 (2009) [arXiv:0812.4016 [hep$\mathrm{ph}]$.

[50] A. Arvanitaki, S. Dimopoulos, S. Dubovsky, P. W. Graham, R. Harnik and S. Rajendran, arXiv:0904.2789 [hep-ph].

[51] W. Buchmuller, A. Ibarra, T. Shindou, F. Takayama and D. Tran, arXiv:0906.1187 [hep-ph].

[52] A. Ibarra, D. Tran and C. Weniger, arXiv:0906.1571 [hep-ph].

[53] C. H. Chen, C. Q. Geng and D. V. Zhuridov, arXiv:0906.1646 [hep-ph].

[54] X. G. He, arXiv:0908.2908 [hep-ph].

[55] B. Katz, K. Blum and E. Waxman, arXiv:0907.1686 [astro-ph.HE].

[56] G. Kane, R. Lu and S. Watson, arXiv:0906.4765 [astroph.HE].

[57] O. Adriani et al., Phys. Rev. Lett. 102, 051101 (2009) [arXiv:0810.4994 [astro-ph]].

[58] D. Hooper, A. Stebbins and K. M. Zurek, arXiv:0812.3202 [hep-ph].

[59] P. Brun, T. Delahaye, J. Diemand, S. Profumo and P. Salati, arXiv:0904.0812 [astro-ph.HE].

[60] D. Feldman, Z. Liu and P. Nath, Phys. Rev. D 79, 063509 (2009) [arXiv:0810.5762 [hep-ph]].

[61] M. Ibe, H. Murayama and T. T. Yanagida, Phys. Rev. D 79, 095009 (2009) [arXiv:0812.0072 [hep-ph]].

[62] W. L. Guo and Y. L. Wu, Phys. Rev. D 79, 055012 (2009) [arXiv:0901.1450 [hep-ph]].

[63] X. J. Bi, X. G. He and Q. Yuan, Phys. Lett. B 678, 168 (2009) [arXiv:0903.0122 [hep-ph]].

[64] J. Hisano, S. Matsumoto, M. M. Nojiri and O. Saito, Phys. Rev. D 71, 063528 (2005) [arXiv:hepph/0412403].

[65] J. March-Russell, S. M. West, D. Cumberbatch and D. Hooper, JHEP 0807, 058 (2008) [arXiv:0801.3440 [hep-ph]].

[66] J. B. Dent, S. Dutta and R. J. Scherrer, arXiv:0909.4128 [astro-ph.CO].

[67] J. Zavala, M. Vogelsberger and S. D. M. White, arXiv:0910.5221 [astro-ph.CO].

[68] J. L. Feng, M. Kaplinghat and H. B. Yu, arXiv:0911.0422 [hep-ph].

[69] M. Backovic and J. P. Ralston, arXiv:0910.1113 [hep$\mathrm{ph}$.

[70] L. Bergstrom, Phys. Lett. B 225, 372 (1989).

[71] J. F. Beacom, N. F. Bell and G. Bertone, Phys. Rev. Lett. 94, 171301 (2005) [arXiv:astro-ph/0409403].

[72] A. Birkedal, K. T. Matchev, M. Perelstein and A. Spray, arXiv:hep-ph/0507194.

[73] L. Bergstrom, T. Bringmann, M. Eriksson and 
M. Gustafsson, Phys. Rev. Lett. 94, 131301 (2005) [arXiv:astro-ph/0410359]; E. A. Baltz and L. Bergstrom, Phys. Rev. D 67, 043516 (2003) [arXiv:hep-ph/0211325]; L. Bergstrom, T. Bringmann and J. Edsjo, arXiv:0808.3725 [astro-ph]; D. R. G. Schleicher, S. C. O. Glover, R. Banerjee and R. S. Klessen, arXiv:0809.1523 [astro-ph]; V. Barger, Y. Gao, W. Y. Keung and D. Marfatia, arXiv:0906.3009 [hep-ph].

[74] N. F. Bell and T. D. Jacques, Phys. Rev. D 79, 043507 (2009) [arXiv:0811.0821 [astro-ph]].

[75] R. M. Crocker, D. I. Jones, F. Melia, J. Ott, \& R. J. Protheroe, Nature, 463, 65 (2010)

[76] T. N. LaRosa, C. L. Brogan, S. N. Shore, T. J. Lazio, N. E. Kassim and M. E. Nord, Astrophys. J. 626, L23 (2005) [arXiv:astro-ph/0505244].

[77] K. Ferrière, W. Gillard and P. Jean, Astron. Astrophys. 467, 611 (2007).

[78] W. Reich, P. Reich, E. Fuerst, Astron. Astrophys. Supp. 83, 539 (1990).

[79] A. R. Duncan et al., Mon. Not. Roy. Astron. Soc. 277, 36 (1995).

[80] W. Reich, E. Fuerst, P. Steffen, K. Reif, C. G. T. Haslam, Astron. Astrophys. Supp. 58, 197 (1984).

[81] T. Handa et al., Proc. Astron. Soc. Jap. 39, 709 (1987).

[82] G. Bertone, M. Cirelli, A. Strumia and M. Taoso, JCAP 0903, 009 (2009) [arXiv:0811.3744 [astro-ph]].

[83] F. A. Aharonian et al., Nature 439, 695 (2006).

[84] S. D. Hunter et al., Astrophys. J. 481, 205 (1997).

[85] Mack, G. D., Jacques, T. D., Beacom, J. F., Bell, N. F., Yü ksel, H. 2008, Phys. Rev. D , 78, 063542

[86] T. A. D. Paglione, J. M. Jackson, A. D. Bolatto and M. H. Heyer, Astrophys. J. 493, 680 (1998).

[87] R. M. Crocker et al., Astrophys. J. 666, 934 (2007) [arXiv:astro-ph/0702045].

[88] R. J. Protheroe, J. Ott, R. D. Ekers, D. I. Jones and R. M. Crocker, Mon. Not. Roy. Astron. Soc. 390, 683 (2008) [arXiv:0807.0127 [astro-ph]].

[89] S. Gabici, F. Aharonian and P. Blasi, Astrophys. Space Sci. 309, 365 (2007) [arXiv:astro-ph/0610032].

[90] J. C. Higdon, R. E. Lingenfelter and R. E. Rothschild, Astrophys. J. 698, 350 (2009) [arXiv:0711.3008 [astro$\mathrm{ph}]$.

[91] S. Roy, A. Pramesh Rao and R. Subrahmanyan, Astron. Astrophys. 478, 435 (2008).

[92] D. N. Spergel and L. Blitz, Nature 357, 665 (1992).

[93] K. Koyama, H. Awaki, H. Kunieda, S. Takano and Y. Tawara, Nature 339, 603 (1989).

[94] W. R. Webber, Astrophys. J. 506, 329 (1998).

[95] M. Revnivtsev, S. Sazonov, E. Churazov, W. Forman, A. Vikhlinin and R. Sunyaev, Nature 458, 1142 (2009).

[96] F. Yusef-Zadeh and M. Morris, Astronom. J. 94, 1178 (1987).

[97] M. Morris and F. Yusef-Zadeh, Astrophys. J. 343, L703 (1989).

[98] M. Morris, arXiv:astro-ph/0701050.

[99] J. F. Navarro, C. S. Frenk and S. D. M. White, Astrophys. J. 490 (1997) 493 [arXiv:astro-ph/9611107].

[100] J. N. Bahcall and R. M. Soneira, Astrophys. J. Suppl.
44, 73 (1980).

[101] P. Gondolo, J. Edsjo, P. Ullio, L. Bergstrom, M. Schelke and E. A. Baltz, JCAP 0407 (2004) 008 [arXiv:astroph/0406204].

[102] E. Borriello, A. Cuoco and G. Miele, Phys. Rev. D 79, 023518 (2009) [arXiv:0809.2990 [astro-ph]].

[103] G. B. Rybicki and A. P. Lightman, "Radiative Processes in Astrophysics", John Wiley 6 Sons: New York (1979).

[104] M. S. Longair, "High-energy astrophysics", ISBN 0521387736. Cambridge, UK: Univ. Pr. (March 1992) $436 \mathrm{p}$.

[105] T. A. Porter, I. V. Moskalenko and A. W. Strong, Astrophys. J. 648, L29 (2006) [arXiv:astro-ph/0607344].

[106] S. J. Sturner et al., Astrophys. J. 490, 619 (1997).

[107] Bergström, L., Ullio, P., \& Buckley, J. H. 1998, Astroparticle Physics, 9, 137

[108] D. Hooper, Phys. Rev. D 77 (2008) 123523 [arXiv:0801.4378 [hep-ph]].

[109] D. N. Spergel et al. [WMAP Collaboration], Astrophys. J. Suppl. 170, 377 (2007) [arXiv:astro-ph/0603449].

[110] D. Hooper, D. P. Finkbeiner and G. Dobler, Phys. Rev. D 76, 083012 (2007) [arXiv:0705.3655 [astro-ph]].

[111] P. Grajek, G. Kane, D. J. Phalen, A. Pierce and S. Watson, [arXiv:0807.1508 [hep-ph]].

[112] M. Regis and P. Ullio, Phys. Rev. D 78 (2008) 043505 [arXiv:0802.0234 [hep-ph]].

[113] M. Pato, L. Pieri and G. Bertone, Phys. Rev. D 80, 103510 (2009) [arXiv:0905.0372 [astro-ph.HE]].

[114] R. Catena, N. Fornengo, M. Pato, L. Pieri and A. Masiero, arXiv:0912.4421 [astro-ph.CO].

[115] J. F. Beacom, N. F. Bell and G. D. Mack, Phys. Rev. Lett. 99, 231301 (2007) [arXiv:astro-ph/0608090].

[116] H. Yuksel, S. Horiuchi, J. F. Beacom and S. Ando, Phys. Rev. D 76, 123506 (2007) [arXiv:0707.0196 [astro-ph]].

[117] M. Kachelriess and P. D. Serpico, Phys. Rev. D 76, 063516 (2007) [arXiv:0707.0209 [hep-ph]]. See also N. F. Bell, J. B. Dent, T. D. Jacques and T. J. Weiler, Phys. Rev. D 78, 083540 (2008) [arXiv:0805.3423 [hepph]]; J. B. Dent, R. J. Scherrer and T. J. Weiler, Phys. Rev. D 78, 063509 (2008) [arXiv:0806.0370 [astro-ph]].

[118] L. Hui, Phys. Rev. Lett. 86, 3467 (2001) [arXiv:astro$\mathrm{ph} / 0102349]$.

[119] A. V. Belikov and D. Hooper, arXiv:0904.1210 [hep-ph].

[120] S. Galli, F. Iocco, G. Bertone and A. Melchiorri, Phys. Rev. D 80, 023505 (2009) [arXiv:0905.0003 [astroph.CO]].

[121] G. Huetsi, A. Hektor and M. Raidal, arXiv:0906.4550 [astro-ph.CO].

[122] M. Cirelli, F. Iocco and P. Panci, arXiv:0907.0719 [astro-ph.CO].

[123] T. R. Slatyer, N. Padmanabhan and D. P. Finkbeiner, arXiv:0906.1197 [astro-ph.CO].

[124] P. Meade, M. Papucci, A. Strumia and T. Volansky, arXiv:0905.0480 [hep-ph].

[125] I. Cholis and N. Weiner, arXiv:0911.4954 [astro-ph.HE].

[126] E. Wommer, F. Melia and M. Fatuzzo, Mon. Not. Roy. Astron. Soc. 387, 987 (2008) [arXiv:0804.3111 [astro$\mathrm{ph}]$. 